\title{
EXPERIMENTAL STUDY ON THE VIABILITY OF DECENTRALIZED RAILWAY TRAFFIC MANAGEMENT
}

\author{
QUENTIN PERRACHON ${ }^{1}$, RÉMY CHEVRIER ${ }^{1} \&$ PAOLA PELLEGRINI $^{2}$ \\ ${ }^{1}$ SNCF Innovation and Research, France \\ ${ }^{2}$ COSYS-LEOST, University Gustave Eiffel, IFSTTAR, University Lille, France
}

\begin{abstract}
In this paper we propose an experimental study aimed at assessing a new paradigm for railway traffic management. In current practice and academic state of the art, train priority decisions in the case of perturbation are made centrally. In this work, train traffic is modelled as a multi-agent system in which trains are decision makers. We implement a microscopic simulator for assessing the viability of this decentralized traffic management and for comparing it with the centralized one. Our results show that it is indeed possible to let trains decide their own priority and it is possible to achieve results which are comparable to simple centralized approaches in terms of passenger travel time in the mass-transit area of Paris, France.

Keywords: railway dispatching, decentralisation, multi-agent system.
\end{abstract}

\section{INTRODUCTION}

Railway traffic management (RTM) follows a centralized approach in which dispatchers regulate trains from an operational control centre. Their role is to manage trains with a global vision of traffic, current and future, by anticipating conflicts. In conflicts, one or more trains have to slow down because the track section they need to traverse is occupied. Dispatchers' decisions lead to the interlock of itineraries for trains to pass.

Thanks to novel technologies in the fields of, for example, communication, computing and artificial intelligence, it is now possible to reconsider the way to manage traffic. In relation with the recent works on autonomous vehicles in road traffic and the SNCF autonomous train project, our idea is to not only distribute RTM but also to decentralize dispatching decisions directly on trains.

This research theme is quite unexplored [1]. Very few papers in the literature focus on non-centralized approaches [2]-[4]. The first paper proposing a decentralized approach [5] describes a model in which the train behaviours are conditioned by the impact of their traveling on other trains. However, many details are missing.

Globally, the few existing approaches are rather focused on the distribution of the operational decision centres, i.e., on the organization of physical decision centres in the network, than on the decentralization of the decision-making. Compared to the distributed approach which keeps local decision centres, the decentralized one is fundamentally different because of the change of paradigm: there is no more "decision centre". Decisions are made by trains themselves, which are thus no more passive executors but become active actors of the system.

In this paper, we present a study on this very prospective research theme. We bring results obtained on simulations inspired from real existing lines in the Paris area: the L and RER A lines.

The rest of the paper is organized as follows. Section 2 details the rationale and the objective of this study. Section 3 describes the characteristics of the railway system which we consider in the analysis, and Section 4 the ones of the multi-agent system we use for representing traffic. Sections 5 and 6 report the simulation set-up and results we achieve. Section 7 concludes the paper. 


\section{RATIONALE AND OBJECTIVES}

With regards to the evolution of computer science or other fields, there is a progressive evolution from centralized approaches to decentralized ones. This evolution responds to needs such as robustness face to the risks of system failures and resource availability.

Transposed to the railway system, these characteristics incite us to reconsider the way of managing traffic and to introduce changes within it. For that, AI and communication technologies make it possible to change the role of trains from passive, i.e., receiving and executing orders, to active.

As new research theme, a lot of underlying research topics will have to be investigated. Our objective is to make a first step towards the experimental assessment of the fully decentralized RTM concept, in terms of viability and ability to reach results of comparable quality to the centralized ones. As a first step, we focus on conflict resolution at convergences in the network and on the assessment of the impact of communication disruptions.

\section{MODELING OF THE RAILWAY SYSTEM}

We model train traffic as a multi-agent system. To do so, train-agents need a specific railway environment, that commercial railway simulators do not offer. Then, we develop a simulator for this study. With the representation of track sections and the block signals, it is microscopic and simulates actual train protection systems.

Given that the accuracy of the physical models used to represent train runs is not necessary to prove the feasibility of the decentralized approach, we choose a simplified railway modelling. We use the motion formula with constant acceleration and braking. Hence, we calculate speed profiles of train runs considering distance and speed as functions of time. Tracks are divided into sets of block sections. A block is delimited by two signals and defined by a length and a speed limit. To ease computations and make them faster, parameters such as gradient or curve radius are not taken into account in the modelling.

Blocks are delimited by three-aspect signals which are very common in railways: (i) freeway (green): the train can run at the authorized maximum speed; (ii) closed (red): the train has to stop in front of the signal; and (iii) warning (yellow): the train driver is notified that the next signal is closed (red) and has to follow a speed control curve to be able to stop at the next signal if it is still closed when the train arrives in front of it.

Hence, signalling separates trains and protects specific areas which may see the emergence of conflicts such as switches in junction areas. Indeed, when a train enters a block, the latter is locked and all signals protecting the corresponding track section turn to red.

The example in Fig. 1 shows that train A is late whereas train B is on time, and they arrive at the junction simultaneously. To respect the planned order, train B has to stop before the block including the switch to let train A pass. Hence, also train B will suffer a delay for the rest of its route.

To match the reality of our case study, we model the French KVB device (beacon-based speed control) [6].

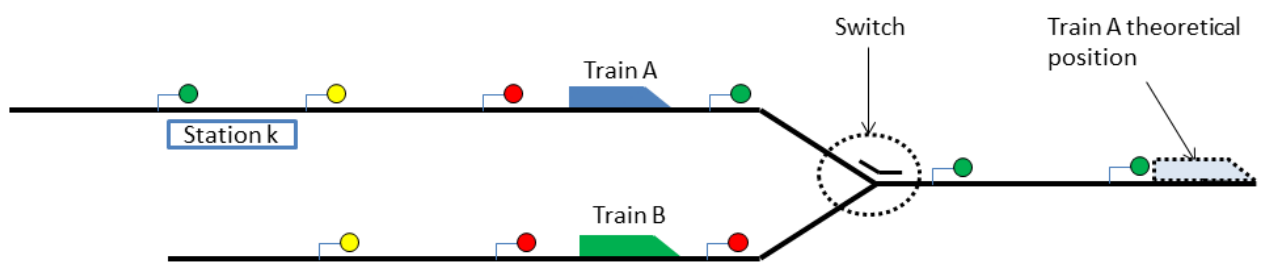

Figure 1: Conflict occurring with the simultaneous arrival of two trains at a junction. 


\section{MODELING OF TRAIN-AGENTS}

In this section, we describe the system we put in place to assess the performance of decentralized RTM. In particular, each train is an agent. Each train is defined by a timetable which gives the departure times from the stations it serves and a utility function is associated to possible delays. The timetable is conflict-free, but conflicts may emerge due to perturbations, especially at junctions as explained in Section 3.

When conflicts emerge, train-agents do not have this global vision of the traffic situation and must coordinate to properly schedule their arrival at the junction. In our modelling, trains know the characteristics of the infrastructure on which they run and the timetable of neighbour trains, and do trajectory projections from their current position.

When a train detects a conflict, it informs the neighbour trains. Then, they decide how to solve the conflict and schedule their passing order by exchanging their utility values regularly. We consider different decision-making strategies as explained in Section 5. Along the exchanges, the trains can possibly update their utility values. The final decision is made at the latest when the train which has to pass first according to the current passing order reaches the junction (Fig. 2).

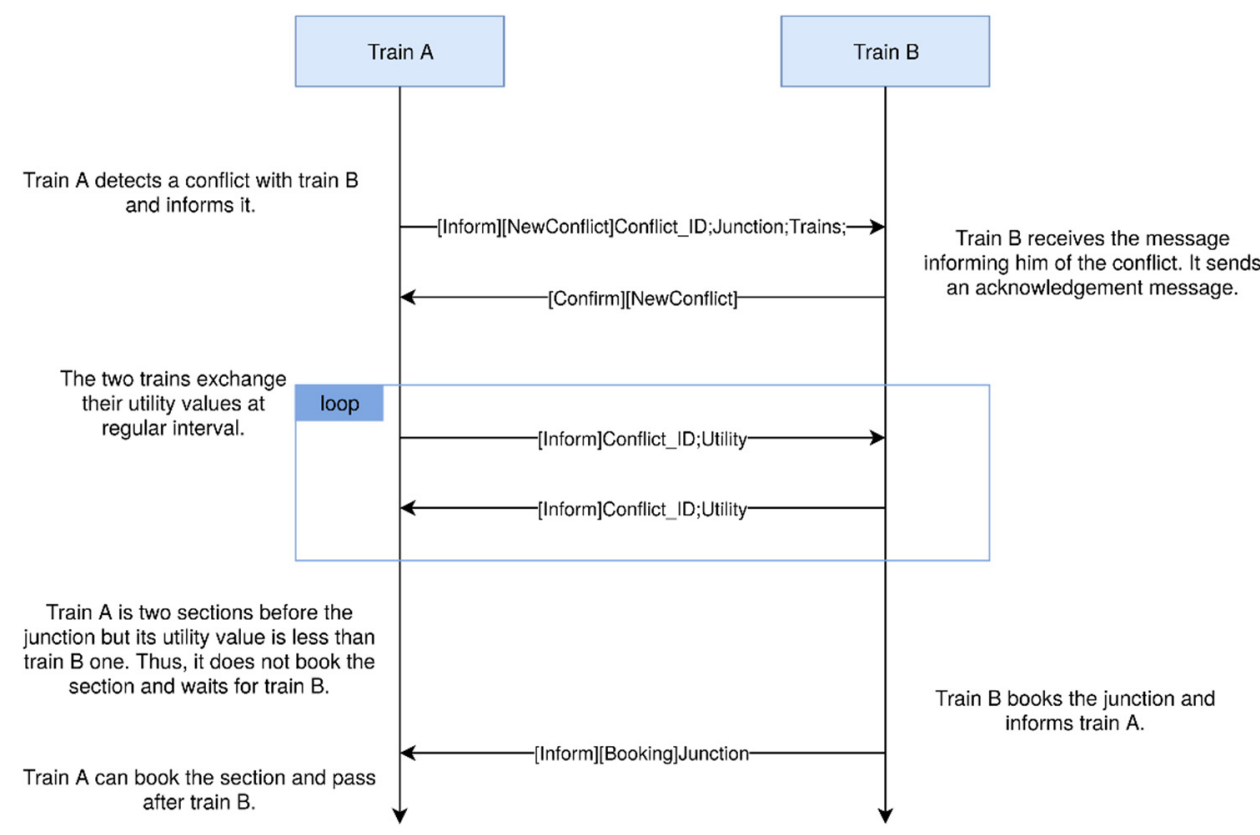

Figure 2: Conflict solution procedure.

\section{EXPERIMENTAL SET-UP}

In this section, we describe the instances on which we assess the decentralized approach. We also explain the criteria chosen to evaluate and compare the decentralized and the centralized approaches, as well as the decision making strategies considered.

At the end of a simulation run, we assess the traffic in terms of passenger travel time. In particular, we consider the ratio between the actual travel time and the planned one, i.e., the minimum time necessary to go from a station to another in nominal traffic. We consider the total of these times over all passengers. 


\subsection{Instances}

We consider a network inspired from a perimeter operated by both SNCF Transilien and RATP, the two main operators in the Paris area: the western RER A and L lines, as depicted in Fig. 3. The SNCF part of the line has two terminal stations: Cergy and Poissy. Trains run towards Paris through the Nanterre-Préfecture station, from which the line is operated by RATP. Line L also starts from Cergy station and shares its infrastructure with the RER A branch from Cergy to Maisons-Laffitte and with two RER A branches from Maisons-Laffitte to the Nanterre divergence. From this divergence, the L trains run towards the Paris SaintLazare station (terminus). The third branch of the RER A line, operated by RATP, departs from Saint-Germain-en-Laye and joins the other branches before the Nanterre-Préfecture station.

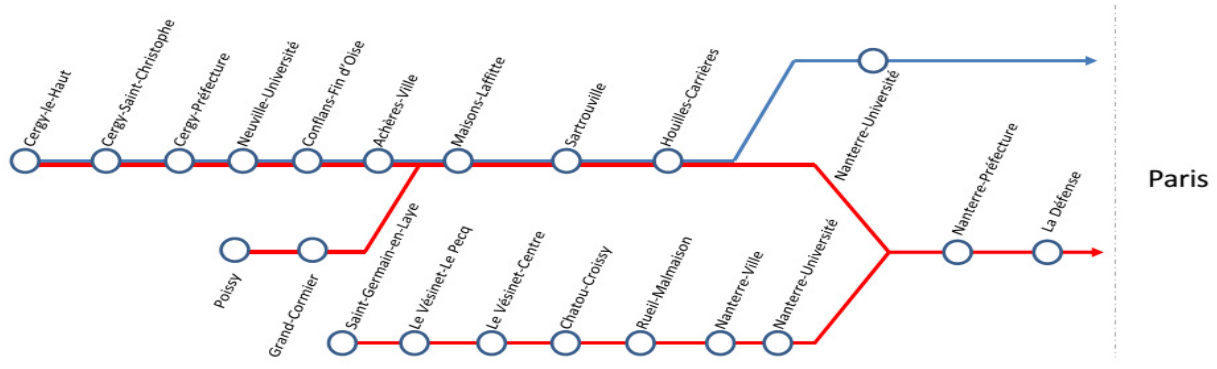

Figure 3: Schematic description of the western RER A (red) and L (blue) lines.

In the morning peak hours, the nominal timetable is cyclic and periodic and plans (per hour): $6 \mathrm{~L}$ trains departing from Cergy-Le-Haut towards Nanterre-Université; 6 RER A trains departing from Cergy-Le-Haut; 6 RER A trains departing from Poissy; 6 RER A trains departing from Saint-Germain-en-Laye. One train from each line and branch departs every ten minutes and the trains gather in a common flow between Maisons-Laffitte and HouillesCarrières stations, after which line L goes to Nanterre-Université and RER A to NanterrePréfecture. Before reaching this station, the traffic flow of the three RER A branches merge. It has to be noted that the RER A trains from Cergy are not omnibus and do not stop neither at Maisons-Laffite nor at Houilles-Carrières stations.

All other trains are omnibus. All stops theoretically last 30 seconds, but they often end up to be longer due to large passenger flows. This is a frequent source of perturbation. The infrastructure topology includes double tracks and multiple platforms in some stations, allowing trains to overtake others if necessary. Trains traveling in opposite directions never share tracks, hence we consider only the ones going to Paris.

We consider three 6-hour simulation scenarios. The simulations differ in the passengers' arrival pattern. In the proportional simulation the number of passengers uniformly increases at all stations at peak time. The passenger ratio is constant through lines, and the trains originating at Cergy are more crowded that those coming from Poissy, since the former cross more stations. In the inverse simulation, the number of passengers is inversely proportional to the number of stations traversed: trains from Poissy are more crowded than those from Cergy. Finally, in the average simulation the number of passengers from both origins is in average the same. In these simulations, we are particularly interested in observing the interaction between trains originating in Cergy and Poissy: the junction between the two 
branches has a strong impact on the rest of the line, due in particular to the different services performed. Passengers arrive at stations in a continuous flow and try alight in the first train which stops, provided that capacity is available. The destination station of each passenger is fixed in coherence with the observed demand on the lines.

\subsection{Traffic management strategies}

To define the train-agents' decision making, first we compare the system performance when traffic is managed according to three simple strategies: on-time, according to which on-time trains have priority over delayed ones; delay, according to which, on the contrary, delayed trains have priority; passengers, according to which train priorities are proportional to the number of on-board passengers when the conflict is detected. Indeed, being the strategy decentralized, only the trains directly involved in a conflict are considered.

Then, to make a step forward toward the definition of a finer strategy, we consider the implication of the choices after the conflict. To do so, each train makes a projection of the delay it will generate for all trains in its neighbourhood if it passes first, taking into account all trains with which it has a conflict. This delay is typically named secondary delay in the railway community. Since it is the train perspective, the projection can be local and no computational issues emerge. Considering this generated delay, we introduce two more strategies: secondary, according to which the train generating the smallest secondary delay has priority; weighted secondary, according to which the train which generate the smallest secondary delay weighted by the number of passengers on each train has priority.

For comparison, we consider two centralized traffic management strategies: $f c f s$ (firstcome-first-served), in which the fist train claiming the use of the junction passes first, and plan, in which the train priority follows the original timetable disregarding delays. The latter strategy is the one currently implemented on this line. Finally, we take into account the centralized strategy: we use the same criteria as the best decentralized strategy, but the decision is made centrally. The difference between decentralized and centralized strategies is the larger scope of the latter, in which we do not consider only the immediate neighbourhood of each train in the utility assessment of a decision.

As an example of the difference between the centralized and decentralized strategy, we consider the following situation in the network shown in Fig. 3. One train departing from Cergy arrives at the junction together with one departing from Poissy. Another train departing from Cergy follows closely. In a decentralized traffic management strategy, the first two trains will detect their conflict and they will assess the impact of the two possible entry orders in the common track. The order with the lower impact will be privileged. If the train from Cergy passes second, it may possibly penalize the following train, too, but this is not considered given the local nature of the decision. In a centralized strategy, instead, all trains are considered and a globally good solution is sought. Indeed, this comes at the cost of a higher computational complexity of the centralised case.

\section{SIMULATION RESULTS}

In this section we report the results of the simulations performed. We start by comparing different decentralized traffic management strategies. Then we select the best performing one and we compare it with centralized ones. 


\subsection{Decentralised strategies}

Fig. 4 reports the comparison of the proportional, inverse and average simulations described in Section 5.2 for each simulation scenario depicted in Section 5.1. Specifically, we analyse the evolution over time of the ratio between the actual total travel time and the planned one. As it is evident, the passengers strategy appears effective in the first two simulations. This was indeed expectable, since a train delay impacts the total travel time proportionally to its number of passengers. Instead, if trains transport equivalent numbers of passengers (average simulation), this strategy becomes less effective. For what concerns the two other strategies, on-time and delay, their relative performance changes in the different simulations. This is due, again, to the different numbers of passenger in the trains originating from Cergy and Poissy and to the characteristics of the infrastructure. Indeed, as it can be seen in Fig. 3, the line branch from Cergy is longer than the one from Poissy. Trains coming from the former face more perturbations and cross more stations than the others. Hence, they get to the junction with higher delay and are penalized. When the passenger flow is higher from Cergy in the proportional simulation, the delay strategy prioritizing trains with higher delay implies that these most crowded trains are favoured. Thus, the total travel time is lower than in the case in which the on-time strategy is applied and the trains from Poissy have priority. The opposite holds in the inverse simulation, as expected.

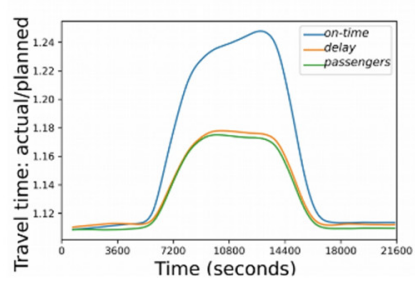

(a)

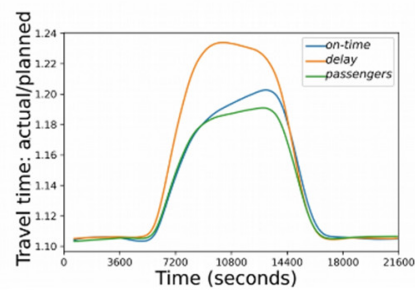

(b)

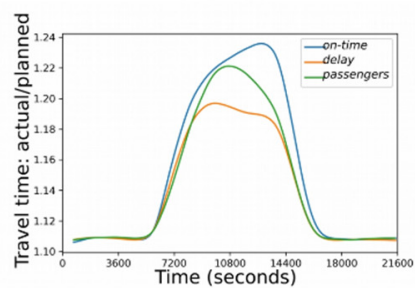

(c)

Figure 4: Assessment of traffic management strategies in the proportional (a); inverse (b); and average (c) simulations: on-time, delay and passengers strategies.

This analysis shows that these three simple strategies are too naive: if the number of passengers does not make obvious the appropriate priority to affect to trains, these three strategies are not capable of properly capturing the system performance associated to traffic management choices.

Hence, we compare the performance of the two finer secondary and weighted secondary strategies with the passengers one, which had the best results in the first round of experiments, on the three simulation scenarios. The results are shown in Fig. 5.

As it could be expected, the weighted secondary strategy appears the best performing in almost every case. Only in the average simulation, the secondary strategy performs slightly better at peak time. The fact that weighted secondary does not always minimize the actual total travel time depends on the lack of knowledge on the passengers' destination when the secondary delay projection is made. Indeed, if there is at least one stop between the junction where the conflict emerges and the station where the delay is suffered, some passengers may get on and off the train and this will slightly change the impact of the traffic management choice with respect to the expectation. Indeed, it may be possible to use information on 


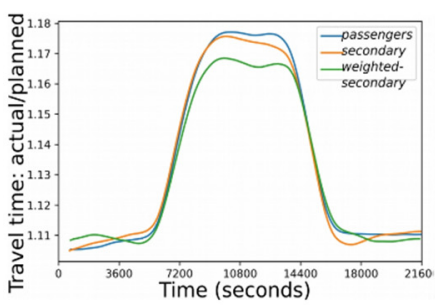

(a)

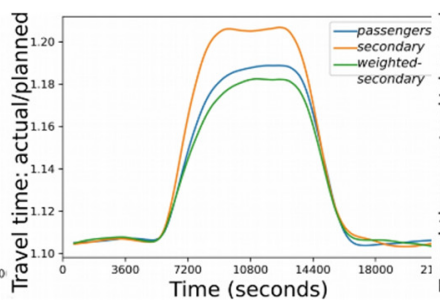

(b)

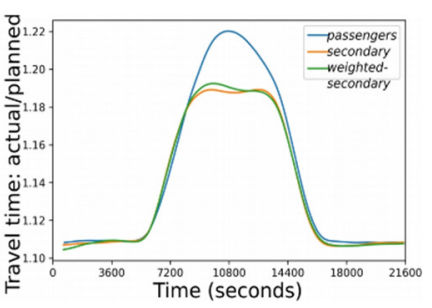

(c)

Figure 5: Assessment of traffic management strategies in the proportional (a); inverse (b); and average (c) simulations: passengers, secondary and weighted secondary strategies.

passengers' trips if available, for example in case the train tickets include destination data. However, this piece of information is not often available, as in our case study. Another option may consist in the inclusion of an analysis of the infrastructure and of the services planned when a train needs to assess the secondary delay it would generate by passing first. Based on this analysis, an educated guess on the number of passengers suffering this secondary delay may replace the straight computation of the weighted secondary strategy. Nonetheless, this would complicate quite significantly the computation and we consider the performance of the proposed strategy good enough not to justify the additional complication.

In the following, we will consider the weighted secondary strategy and we will simply name it decentralized.

\subsection{Centralized vs decentralized approach}

In this section, we report the experimental analysis which we obtain by comparing the decentralized strategy with two centralized ones mentioned in Section 5.2. We consider the proportional simulation presented in Section 5.1. Fig. 6(a) reports these results. They show that the decentralized strategy achieves the best performance in this comparison. The plan one is quite competitive with it, but especially at peak time the difference is quite remarkable. Indeed, the $f c f s$ strategy is definitely outperformed.

In Fig. 6(b), we show the results of a further comparison: we add to the decentralized and the plan strategy the results achieved with the centralized one.

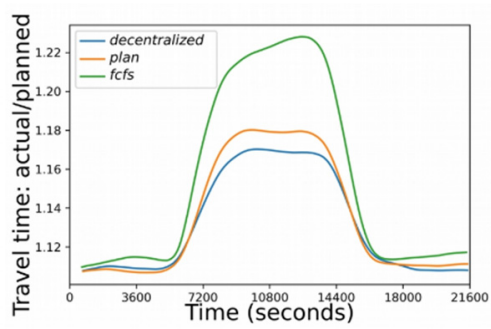

(a)

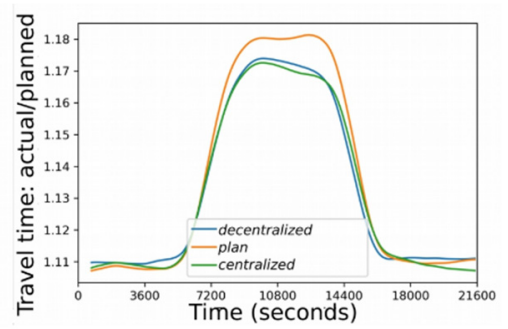

(b)

Figure 6: Assessment of centralized and decentralized traffic management strategies.

(a) Decentralized, fcfs and plan in the proportional simulation; and

(b) Decentralized, plan and centralized in the average simulation. 
As it can be seen, the difference between these two strategies is very small. In our eyes, these results show that managing traffic in a decentralized way is a viable option, which does not significantly worsen the performance from the system perspective.

\section{CONCLUSIONS AND PERSPECTIVES}

In this paper, we proposed an experimental study aiming to be a first step towards the assessment of the feasibility of a new paradigm for railway traffic management. In this new paradigm, decisions are made in a decentralized framework: traffic is modeled as a multiagent system. We implemented a simulator to perform this study.

We tested different decentralized strategies for traffic management, and we compared them with centralized ones. The simulation results show that traffic is indeed manageable in a decentralized way, without any remarkable loss of service quality. We measured service quality as the ratio between the total passengers' travel time computed after simulation and the theoretically possible one.

In future research, we will study more articulated decentralized strategies, focusing on state of the art approaches for decision making in multi-agent systems. These strategies will allow assessing the performance of the new paradigm on more complex networks with mixed traffic and different traffic characteristics. On these networks, we will compare the decentralized system with a centralized one in which traffic is managed in an optimized fashion, using well performing algorithms existing in the literature. Moreover, we will assess the impact of communication failures on the performance of the system, to quantify the difference in the robustness of centralized and decentralized RTM.

\section{ACKNOWLEDGEMENT}

The work of Paola Pellegrini was partially funded by the CPER ELSAT2020 project cofinanced by the European Union with the European Regional Development Fund, the French state and the Hauts-de-France Region Council.

\section{REFERENCES}

[1] Marcelli, E. \& Pellegrini, P., Literature review toward decentralized railway traffic management. IEEE Intelligent Transportation Systems Magazine, 2020.

[2] Haxthausen, A.E. \& Peleska, J., Formal development and verification of a distributed railway control system. IEEE Transactions on Software Engineering, 8, pp. 687-701, 2000.

[3] Parkes, D.C. \& Ungar, L.H., An auction-based method for decentralised train scheduling. Proceedings of the 5th International Conference on Autonomous Agents, pp. 43-50, 2001.

[4] Vernazza, G. \& Zunino, R., A distributed intelligence methodology for railway traffic control. IEEE Transactions on Vehicular Technology, 39(3), pp. 263-270, 1990.

[5] Cui, Y., Martin, U. \& Liang, J., Decentralized, autonomous train dispatching using swarm intelligence in railway operations and control. 7th International Conference on Railway Operations Modelling and Analysis, RailLille2017, pp. 521-540, 2017.

[6] Chevrier, R. \& Ramond, F., Improving the mass-transit flow by train-holding in station with a simple and efficient heuristic. International Journal of Transport Development and Integration, 2(4), pp. 330-341, 2018. 